\title{
The Coding of Images by the Retina
}

\author{
M. Imbert, Paris
}

(Institut des Neurosciences, Université Pierre et Marie Curie)

In his article: "From the Photon to the Neuronal Signal" [Europhys. News 16 (1985) 5], Marc Chabre discussed how the photoreceptor cells convert photon fluxes into electrical signals amenable to neuronal analysis. This early stage is followed by complex neuronal processing, the first beginnings of which develop in the distal part of the retina itself, and then continue with the generation of nerve impulses in the proximal part which transmits to the brain a representation of the image.

\section{Basic Structure}

The retina, as illustrated in Fig. 1, is a regularly layered "mat" of receptors and nerve cells covering the back of the eye. In vertebrates, the retina is "inverted", i.e. the photoreceptors are in the furthest layer from the lens with their outer segments containing the visual pigment pointing away from it, so that the light, after passing through the optics of the eye, must penetrate through the entire retina before reaching the outer segment where phototransduction takes place. As Chabre explained: in response to light, the photoisomerization of retinal leads to the closure of $\mathrm{Na}^{+}$channels, which are normally open in the dark, thus producing a hyperpolarization of the entire photoreceptor membrane from -30 to $-60 \mathrm{mV}$. This change initiates the chain of events involved in the processing of visual information.

Three classes of neurone convey information radially from the receptor cells to the ganglion cells. These, linked together by bipolar cells, relay information to the brain. Three other classes of neurone modulate the transmission: the horizontal cell, the amacrine and the interplexiform cell, the last of which carries information from the amacrine to the horizontal and bipolar cells, making a feedback network inside the retina. Interconnections between the retinal neurones are almost exclusively confined to two plexiform layers. The outer layer contains connections between the receptors, bipolar and horizontal cells, and the inner layer, those between the bipolar, amacrine and ganglion cells.

\section{Neurone Coupling Processes}

As everywhere in the nervous system, any given individual neurone establishes very specific connections with other neurones through specialized contact zones, the synapses, whose properties largely determine the functioning of the nervous system. The contacts result from the juxtaposition of the membranes of two different cells and they establish the communication between the cell before (presynaptic) and the cell after (postsynaptic). Neurones that are interconnected in this way form networks whose integrated activity is considered to underlie specific tasks of information processing.

The neurones in a network may be in close proximity or at a great distance from each other. If distant, for example the ganglion cells and the relay cells in the brain, communication occurs via axons, in this case the optic nerve fibres, that conduct discrete action potentials. On the other hand, when the cells are close, as they are within the retina, communication may occur in local circuits by means of graduated electrical signals, conducted passively along the membrane.

But whether close or distant, neurones can exchange signals only at synapses. In some, the coupling is direct, the synapse taking the form of a close apposition of the pre- and postsynaptic membranes forming thereby a "gapjunction" of protein channels through which ions and small molecules can circulate freely. The electrical signal passes without delay, from one cell to the other, and usually in both directions, by mere ionic diffusion. Such electrical coupling is used by the receptor cells which are electrically coupled at their endings, and also by the horizontal cells.

However, the most common synaptic couplings in the two plexiform layers of the retina involve chemical transmission. In the outer plexiform layer, the receptor cell is coupled to both horizontal and bipolar cells through a "ribbon synapse". Several postsynaptic elements penetrate deeply into the presynaptic receptor cell which contains an electron- dense bar, the ribbon, surrounded by clusters of vesicles. Two other types of chemical synapse are found in the plexiform layers, of which one is very similar to the conventional synapse found throughout the brain: a cluster of vesicles in the presynaptic side faces a single postsynaptic process. Horizontal, amacrine, and interplexiform cells make this sort of synapse.

By contrast, the processes of horizontal and amacrine cells are quite different. They may be both pre-and postsynaptic, and both serial and reciprocal. As illustrated in Fig. 1, an amacrine (A) that receives from a bipolar cell (IB) makes a synapse back onto its own presynaptic input.

Finally, a special synaptic contact, most probably chemical in nature, is observed between the receptor cells (in mammals the cones, but both cones and rods in other animals) and the dendrite of some bipolar cells. These junctions have been called superficial, basal, or flat, and the bipolar cell connected by such flat contacts has consequently been termed a flat-bipolar cell, see right part of Fig. 1, FB. There is fairly good evidence that the junctions are truly synaptic.

This outline of the structural organization of the retina clearly shows the complexity underlying visual information processing, but it helps to explain how the ocular image, detected by more than 100 million photoreceptors, can be transmitted to the brain, without loss of information, by trains of impulses through only one million optic fibres. Intracellular recording of individual retinal cells, along with their morphological identification, has allowed us to follow step by step the processes. In some cold-blooded species, because of the large size of their retinal elements, it has been possible to record the intracellular activities and to identify the morphological class of all retinal cells from the receptor to the ganglion cells. In other species, particularly in mammals, such a complete description is not yet available, but extracellular, combined in some instances with intracellular recordings, allow some generalization. 


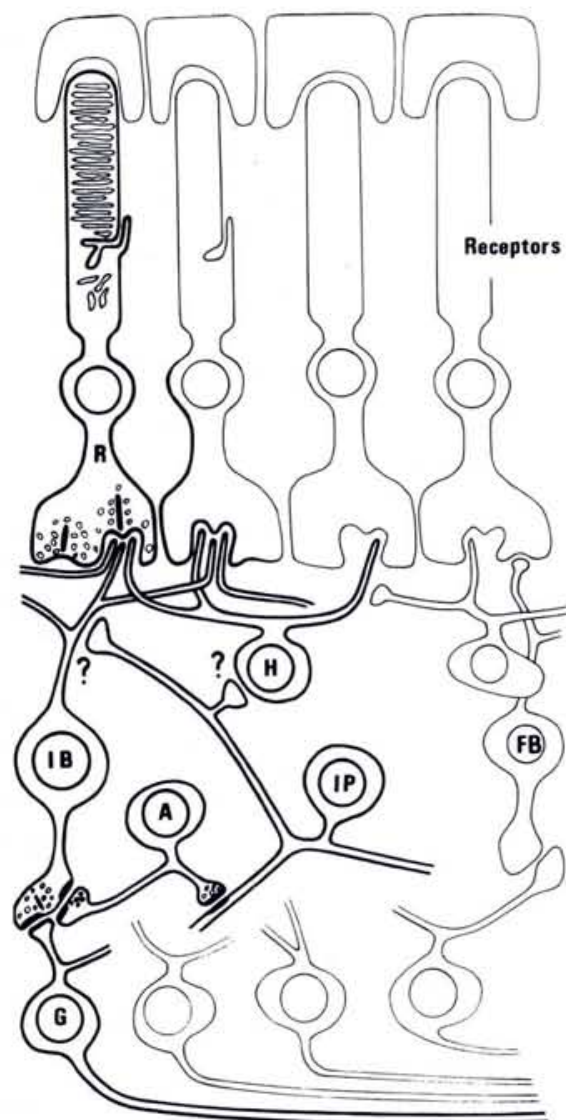

Fig. 1 -Diagram of arrangements of synap tic contacts found in vertebrate retina. $R$ : receptor cell; $H$ : horizontal cell; IB: invaginated bipolar cell; FB: flat bipolar cell; $A$ : amacrine cell; $G$ : ganglion cell; IP: interplexiform cell. See text for details.

\section{Cell Interconnections}

Electrophysiological analysis has permitted the characterization of different types of receptor cell. First there are the rods which have a sensitivity higher than the cones: in the turtle retina for example, rods produce a hyperpolarization of about $1 \mathrm{mV}$ photoisomerization $\left(\mathrm{Rh}^{*}\right)$, the cones only about $25 \mu \mathrm{V}$. Second, the cones, in species with colour vision, have sensitivities which are only maximal within a restricted region of the visible spectrum.

The classical notion that receptor cells are independent of each other, and "signal" only the light which directly impinges on them, must be discarded. In lower vertebrates, rods and cones are interconnected by gap junctions between cells of the same class. The amplitude of the response in one receptor cell is increased when neighbouring receptors are also illuminated. This "spatial summation" results from electrical coupling between receptor cells. However, since the electrical signal, which is passively propagated, is attenuated with distance, the summation effect is restricted to an area of about $50-100 \mu \mathrm{m}$ in diameter, an area which is considerably larger than the cross-section of a photoreceptor. In higher vertebrates, particularly in cats and monkeys, rods are not electrically coupled, although a few gap junctions between rods and cones have been identified in the outer plexiform layer of the cat retina. In some cases, cone receptors may interact through synaptic connections made by horizontal cells.

There exist different types of horizontal cell with different morphologies, and response properties. Some respond monophasically by hyperpolarizing when nearby receptors are stimulated with monochromatic light of any wavelength, others respond biphasically by hyperpolarising when the illumination is of short wavelength and depolarizing when long. They provide a lateral neuronal pathway for antagonistic interactions between neighbouring retinal areas, either las in the case of the interaction between "green" and "red" cones in the turtle retina) by feedback onto cone receptors, or by contributing to the organization of the receptive fields of bipolar cells (see below).

In mammals there are separate pathways from rods and cones to different classes of bipolar cell: the rod-bipolar and the cone-bipolar. A noteworthy feature of the synaptic transmission at this level is that there are two independent channels from any one receptor, either cone or rod, to two classes of bipolar cell (Fig. 2). Bipolar cells show complex receptive fields. When photoreceptors located in the centre of the receptive field are stimulated, they depolarize cer- tain bipolar cells (ON-centre bipolar cells) and hyperpolarize others (OFFcentre bipolar cells). These bipolar cells therefore respond differently to the neurotransmitter released by the photoreceptor. In the dark, at rest, the membrane potential of cones and rods is more depolarized than those of the majority of neurones $(-30-35 \mathrm{mV})$ which causes their synaptic transmitter to be released continuously. The transmitter, most probably an excitatory aminoacid (glutamic acid?), depolarizes the OFFcentre bipolar cells (as well as the horizontal cells) by opening cation-selective channels in the cell membrane and hyperpolarizes the $\mathrm{ON}$-centre bipolar by closing cation-selective channels.

\section{Receptive Field}

Bipolar cells are synaptically connected not just to their "centre" receptor cells, they also receive information from a part of the ocular image through a larger patch of receptors: those in the centre of the patch are in direct contact with the bipolar cell while those, situated in more peripheral parts, are connected via horizontal cells. This spatial interconnection arrangement, is the basis of the "receptive field" referred to above. This receptive field comprising a centre and a concentric surround, is a key concept for understanding visual processing.

In the case of the bipolar cells, this centre-surround organization is antagonistic. When light falls onto the receptors situated in the centre of a receptive

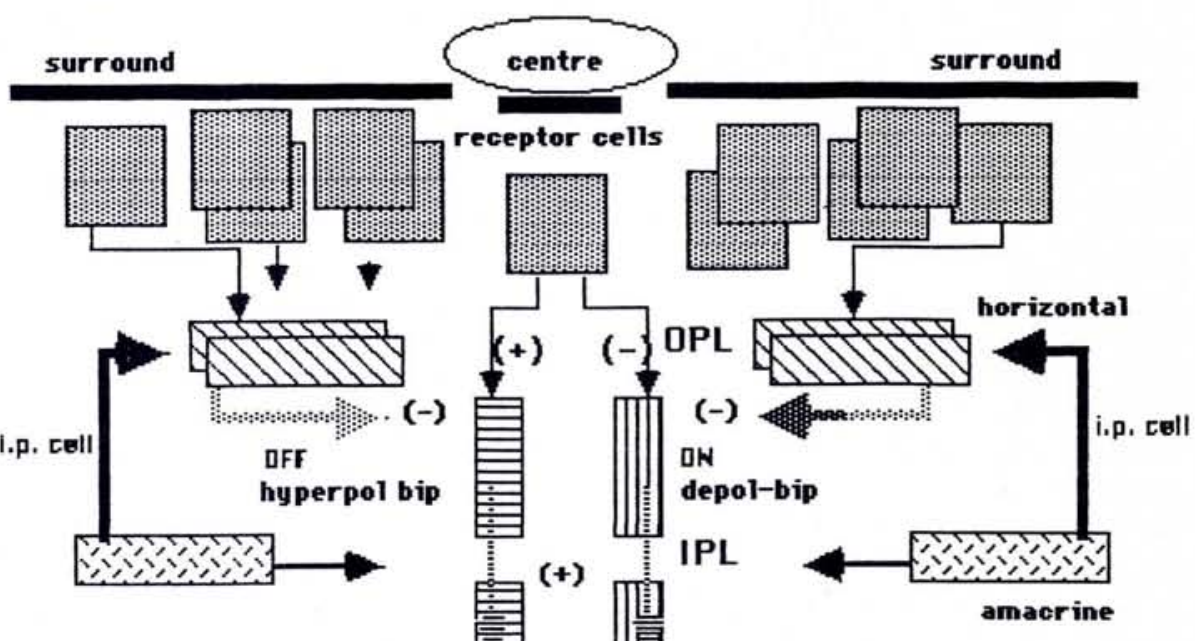

ofF-ganglion

\section{ON-ganglion}

\section{(+): sign-conserving}

$(-)$ : sign-inverting

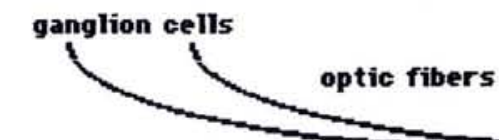

Fig. 2 - Schematic diagram of the functional connections within the vertebrate retina; OPL: outer plexiform layer; IPL: inner plexiform layer; i.p.: interplexiform cell. See text for further details. 
field, the OFF-centre bipolar cells are hyperpolarized, and the $\mathrm{ON}$-centre depolarized. When the surround of the receptive field is stimulated, the $\mathrm{ON}$ centre bipolars become hyperpolarized and the OFF-centre bipolars depolarized. As a result, while stimulation of the centre polarizes the cell in one direction, stimulation of the surround polarizes the same cell in the other direction. Moreover, since the horizontal cells are electrically interconnected, the lateral extent of their influence can be quite large. However, some neurotransmitters or pharmacological agents (dopamine, gamma-aminobutyric acid: GABA) can turn off the electrical coupling between horizontal cells, and thereby modulate the lateral extent of the antagonistic effect of the surround over the centre.

The receptive field arrangement characterizes the functional organization of visual cells in other stations of the visual pathways. As a consequence, a given visual cell monitors a "patch" of visual space through a pathway beginning at the receptor surface by comparing the light falling on two spatially separate sub-populations.

\section{Inner Plexiform Layer}

The bipolar cells are the input to the next processing station, the inner plexiform layer. In this layer, the centre-surround organization of the bipolar receptive field is transferred to the ganglion cells with which they synapse. Here, the amacrine cells, which also make lateral connections, may contribute to the surround for the ganglion cells' receptive field. The synaptic arrangement in the inner plexiform layer is very similar to that in the outer plexiform layer already described.

In most cases, amacrine cells give a transient response at the onset or at the end of visual stimulation which raises the question: how can the sustained ON and OFF responses of the bipolar cells be converted into a transient. A crucial role has been attributed to the reciprocal synapses connecting amacrine to bipolar cells. Such a synapse could locally "turn off" the sustained excitatory action of the bipolar, thus performing a kind of time differentiation. In addition, because of the centre-surround organization of the bipolar cells, the amacrine will be responsive to the space-time dynamic features of the stimulus.

At this point it is worth noting an important difference in the visual processing between the distal and the proximal parts of the retina. In the outer plexiform layer, synaptic actions are carried out through local potentials whose ampli- tudes are a function of the intensity and spatial geometry of the visual stimulus. In contrast, the ganglion and some amacrine cells, generate, when sufficiently depolarized, impulse activity. Whereas in the distal retina all visual information is preprocessed by elements small enough to permit graded electrical signals to be conducted from the receptor site of the cell to the terminal, from the ganglion cells information must be transmitted to the visual centres of the brain by trains of action potentials.

\section{Feedback}

As already noted, there is a strong convergence from photoreceptors to ganglion cells. In such a system it would be very useful if the signals close to the output (in the inner plexiform layer) could automatically control the signals close to the input (in the outer plexiform layer). The interplexiform cells, which receive their synaptic input from amacrine cells, and whose output is situated in the outer plexiform layer, could provide this feedback. In some species, they release dopamine, which is known to alter the electrical coupling between horizontal cells (see above). The effect of such a mechanism would be to modify the lateral extent of the antagonistic effect that the surround exerts on the centre of the receptive field. This would result in an automatic shift of the dynamic range of effectiveness of the receptors, modifying the voltage gain between receptor and bipolar cells. Mechanisms such as this are involved in visual adaptation (q.v.).

\section{Response of the Ganglion Cells}

Since ganglion cells generate impulses, it is possible to monitor their activity by recording the action potentials travelling along a single fibre of the optic nerve (see Fig. 3). Such a recording is relatively easy, and a great number of experiments have been performed in order to characterize different physiological classes of ganglion cells. When the number of action potentials increases, the ganglion cell is considered to be excited, and inhibited when it decreases. In 1953, Stephen Kuffler was able to record impulses emitted by a single ganglion cell in the cat's retina, while stimulating the eye with small spots of light shone in the region of the cell. He showed that the receptive field of the ganglion cells was also organized in two antagonistic circular zones: a centre and a concentric surround. The concentric ganglion cells (as they have been called since that time) can be further divided into two general classes: 1) ON-centre, OFF-surround

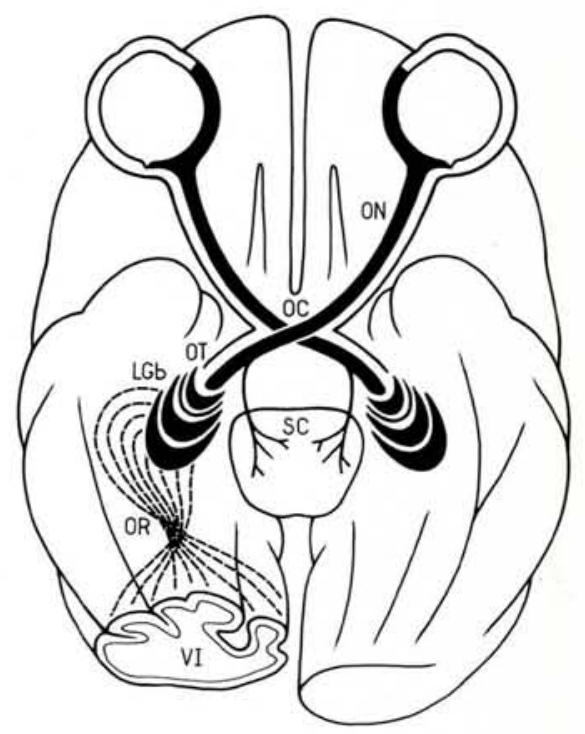

Fig. 3 - Diagram of human visual system. ON: optic nerve; OC: optic chiasm; OT: optic tract; LGb: lateral geniculate body; SC: superior colliculus (not discussed in the text); OR: optic radiations; VI: primary visual cortex.

that increase their discharge when the spot of light is turned on in the centre, and turned off in the surround, and 2) OFF-centre, ON-surround that increase their discharge when the spot is turned off in the centre, and turned on in the surround.

Since the early observations of Kuffler a great diversity of ganglion cell types have been described, beginning with the distinction of two classes of ganglion cells in the cat's eye that were independent of the ON-centre/OFF-centre dichotomy, namely the $\mathrm{X}$-cells and the $\mathrm{Y}$-cells. This distinction is based on the differential responses of the two types of concentric ganglion cells to gratings - regular arrangements of parallel dark and light bars with sinusoidally varying luminance. When the grating moves across the receptive field, at the highest spatial frequencies capable of eliciting a response, the response of an X-cell is always modulated at the drift frequency, while the Y-cells show an unmodulated increase in mean firing rate. When a stationary sinusoidal grating is phase switched at different spatial locations with respect to the centre of the receptive field, the distinction between $X$ - and $Y$ cells can be stated as follows: for the $X$ cells there exists a "null position" where the output of the cell remains unchanged; for $Y$-cells no such position exists.

The $\mathrm{X}$ - and $\mathrm{Y}$-cells may play different roles in perception. At the same retinal position, the $\mathrm{X}$-cells have about three times the spatial resolution of the $Y$ cells. Whereas the Y-cells send an increased train of impulses whenever a pattern is present within their receptive 
field, they are not accurate about its exact location; X-cells on the contrary can give a response when a grating moves from a null position by as little as 0.1 degree of visual angle, corresponding to a change in position of about $10 \mu \mathrm{m}$ on the retina. Moreover, the response of a $Y$ cell to rapid illumination is more transient than that of an X-cell; the Y-cells also respond better to large targets moving at higher velocities than X-cells.

In the cat, but in some other species as well, a few ganglion cells are not classifiable as $\mathrm{X}$ or $\mathrm{Y}$, because their receptive fields are not organized with a centre and an antagonistic surround. Called W-cells, they have axons with a slower conduction velocity and they respond to complex stimuli. Some are excited by an edge of contrast moved in the centre, others only respond to a certain direction of a moving target, irrespective of its contrast. Yet others are colourcoded, they respond with an ON response for blue light and OFF-responses for green and red lights.

This survey reveals that the retina functions as a "small brain inside the eye $^{\prime \prime}$. In spite of its relative simplicity with only five basic types of nerve cell regularly arranged throughout its entire area, the retina performs the most fundamental aspects of visual information processing.

\section{Function Separation}

Different steps of processing may be distinguished, each located at a different level in the retina. The retinal image, blurred by diffraction and aberrations of the optics of the eye, is discretely sampled by the array of approximately $10^{8}$ receptors. In the central part of the retina, the fovea, it has long been known that the spatial resolution limit, or acuity, is set by the spacing between cones, and matches the best resolution of the ocular optics. This high foveal acuity is not significantly degraded by neuronal mechanisms, because foveal ganglion cells have their receptive field centres fed by a single cone. The receptor sampling rate drops rapidly with eccentricity, in keeping with the decline in the optical quality of the ocular image, and the size of the receptive field centre increases. It is clear that the central retina is well adapted to analysing the fine spatial details of objects, while the periphery is designed to detect them. The eye, of course, continually moves, bringing the part of the image to be scrutinized over the fovea. Such a dichotomization between the centre and the periphery of the retina is not limited to spatial resolution: sensitivity also differs, being low in the centre and high in the periphery, and there are differences in colour vision, which is only possible through cones, the only receptor cells present in the fovea. This regional specialization of the retina is particularly well suited to reducing the amount of information necessary to code the image.

Reduction is further achieved by the organization of the receptive fields of the ganglion cells. Each optic fibre is concerned with light falling on a large patch of the image, and reacts to the centresurround difference in illumination: ganglion cells ignore continuity, they only signal something like temporal and spatial derivatives of image luminance. Moreover, in certain species, the receptive field organization is so complex as to allow the coding of specific features, as for example the speed and the direction of a moving target, whatever its contrast, as soon as it is precisely defined in size.

\section{Adaptation}

The visual system works over an enormous $\left(>10^{10}\right)$ dynamic range of scene illumination. However, although the amplitude of the receptor potential increases with the intensity of the stimulus, the intensity-response curve, which is sigmoid, saturates when the stimulus is 3-4 orders of magnitude brighter than the threshold. How adaptation is achieved is not yet completely understood. It is known that when passing from twilight to full daylight, there is a switching between two mechanisms of vision, from the so-called scotopic vision, where rods are involved, to photopic vision, when the rods are saturated and cones, of lesser sensitivity, enter into action. It is also well established that when passing from bright light to darkness, there is a slow recovery of sensitivity involving enzymatic regeneration of the bleached retinal pigments. These processes are important, as are the mechanisms which control the amount of light reaching the visual pigment molecules of the photoreceptors, but they do not account for the rapid changes which allow quasi-instantaneous ajustments to increases in ambient light.

Intracellular recordings from receptor cells have clearly established that a large proportion of adaptation is due to processes occurring in the receptors themselves. Individual photoreceptors can change their sensitivity by a few orders of magnitude by shifting their dynamic operating range towards higher or lower light intensities. In addition, a mechanism close to the receptors must also operate in order to control sensitivity
Fourth International Symposium on Optical and Optoelectronic Applied Sciences and Engineering

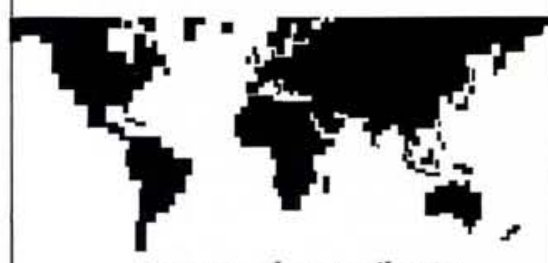

30 March-3 April 1987

The Hague, Netherlands Netherlands Congress Centre

Cooperating Sponsors: Comité Belge d'Optique - DGaO-Deutsche Gesellschaft für angewandte Optik • Optics Division of the European Physical Society $\bullet$ Jet Propulsion Laboratory/California Institute of Technology • Nederlandse Optische Commissie - Promoptica - Société Française d'Optique

Organized by:

ANRT-Association Nationale de la Recherche Technique

SPIE-The International Society for Optical Engineering

With the Organizational Support of Quantoptica Foundation

\section{Topical Meetings}

presenting over 470 technical papers

\section{Equipment Exhibit}

\section{Tutorial Education Program}

\section{Special Plenary Sessions}

Organized by: Hans J. Frankena, Quantoptica Foundation; Hans Tiziani, Optics Division of the European Physical Society; Arnold Dönszelmann, University of Amsterdam

Topics: "Recent Developments in Electron Lithography" "Mass Production of Diffraction Limited Replicated Objective Lenses for Compact Disc Players" "The Production of Optical Fibers for Telecommunication with the PCVD Process" • "High Power Pulsed Gas Lasers"

For full program and exhibit information, or to order Proceedings related to or resulting from these conferences: IN EUROPE contact. ANRTAssociation Nationale de la Recherche Technique, 16, Av. Bugeaud, 75116 Paris, France. Telephone (33.1) 45-53-26-67, Telex 642632. OR contact SPIE, (33.1) 45-53-26-67, Telex 642632. OR contact SPIE, Telephone 2/660-45-11, Telex 25387 AVVAL B.

\section{KL"}

Royal Dutch Airlines Official Air Carrier 
ajustments over the full dynamic range of the input stimulus. Change in the organization of the receptive fields of bipolar cells could bring about adaptation to the background illumination that falls on a large area of the retina, for example by increasing or decreasing the importance of the antagonistic surround: a strong surround illumination results in an increase in the amount of central illumination required for the bipolar cell to respond. Electrical coupling between horizontal cells, and their uncoupling by the interplexiform cells, is particularly well suited to perform such receptive field changes. Since bipolar cells respond with graded potentials, they signal slight deviations from the mean background luminance instead of absolute values. An obvious speculation is that all these mechanisms operate in the outer plexiform layer as an automatic gain control mechanism.

\section{Transmission to the Brain}

In the inner plexiform layer, because of the transient character of the amacrine cell responses, temporal (along with some additional spatial) filtering occurs and finally, the emission from the ganglion cells is in the form of propagated impulses. It is thus tempting to consider the retina as made up of separate channels, overlaid on one another, each transmitting in parallel, several versions of the filtered ocular image. The $X$-, the $Y$-, and the W-cells convey different spatio-temporal aspects of the environment.

An important aspect of processing in the brain is the conservation of this functional segregation up to the cerebral cortex. From the retina, the different classes of optic fibres terminate in separate subregions of the lateral geniculate body (LGb) as seen in Fig. 3, a relay structure interposed between the retina and the occipital cortex. In the primate, ganglion cells, similar to the X-cells of the cat,

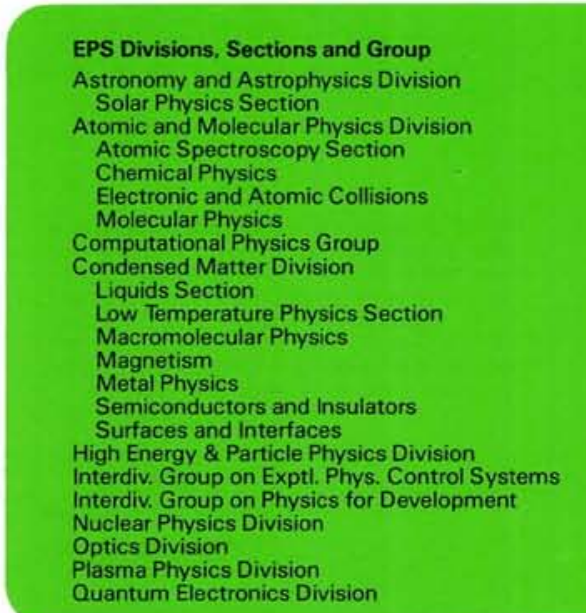

synapse in the dorsal part of the LGb, while cells similar to the $Y$-cells synapse in the ventral part. From LGb to cortex the different channels remain parallel and ramify in separate sub-layers of the input layer of the cortex (layer IV), the first cortical area to receive visual information (see Fig. 4). In the LGb as well as in the cortex, the incoming fibres maintain the topographic retinal distribution of the ganglion cells, thus creating in both structures precise maps of the visual field. Maps, corresponding to each filtered image, are intricately interwoven in layer IV of the cerebral cortex, then distributed to other layers in the same cortical area, before being sent to neighbouring areas, where further processing is performed.

Beside these "parallel" retino-cortical pathways, another important segregation results from the fact that each eye sends axons to both cerebral hemispheres. Optic fibres from the nasal retina cross the optic chiasma and join the fibres from the temporal retina of the other eye in the optic tract (Fig. 3), both groups remaining segregated in different regions of the LGb.

As the number of cells in the primary visual cortex is more than $10^{9}$ (and another $10^{9}$ in the neighbouring visual areas), it is not surprising that a large number of operations, each extracting a specific feature of the environment, take place. Cortical cells are selective to the orientation and size of the stimulus, to the direction of movement, to colour and many other properties. Cell coding for the same orientation are grouped into cortical slabs, perpendicular to the surface, the so-called "orientation columns" (Fig. 4), and most probably, other selective properties of cortical neurones are also grouped in particular pieces of tissue in the cerebral cortex. One must confess that understanding the cortical processing of visual information is not yet as complete as our

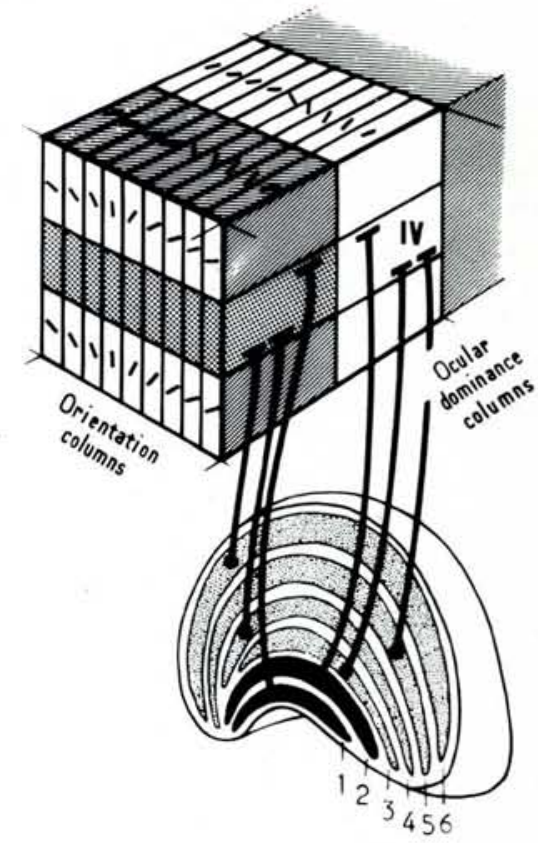

Fig. 4 - Relationship between the lateral geniculate body (LGb) and the visual cortex. The LGb is divided into 6 layers. Layers 1 and 2 receive input from $\gamma$-type ganglion cells and project to the higher part of layer IV in the visual cortex. Layers 3 to 6 receive input from $X$-like ganglion cells and project to the lower part of layer IV in the cortex. Layers 1 . 4 , and 6 receive optic fibres from the nasal controlateral eye, layers 2, 3, and 5 from the ipsilateral temporal retina.

understanding of retinal processing, although much progress has been made in recent years. However, to describe this rapidly advancing field of research would require another article!

\section{BIBLIOGRAPHY}

Barlow H.B., "Understanding Natural Vision", Physical and Biological Processing of Images, Eds. O.J. Braddick and A.C. Sleigh, 2-14 (Springer-Verlag) 1983.

Dowling J.E. and Dubin M.W., "The Vertebrate Retina", Handbook of Physiology, pp: 317-340 Sect. 1, Vol. III, part 1 (American Physiological Society) 1984.

Europhysics News is the official journal of the European Physical Society which comprises 29 National Socie. ties, Academies and Group, about 4000 Individual ties, Academies and Group, about 4000 individual Members and 70 Associate Members. Governing bo-
dies of EPS are the General Meeting. Council and an dies of EPS are the General Meeting, Council and an policy. EPS promotes the collaboration of physicists throughout Europe, organising and harmonising confe. rences and publications, improving physics education, encouraging physics applications, awarding scholarships to sponsored schools in Erice. EPS publishes in addition to Europhys. News, Europhysics Letters (in partnership with national societies), European Journa of Physics (in partnership with The UK Inst. of Phys.) Europhys. Conf. Abs., Europhys, Ed. News. Individual Members receive Europhys. News free of charge (price to insts.: Sw.Fr. 90.-1a), Europhys. Lett. at Sw.Fr, 70.-1a to insts.: Sw.Fr. 90.-fa), Europhys. Lett, at Sw.Fr, 70.-1a
(insts, 595.-1, rebates on many other publications and on conference fees. Annual EPS membership fee for Individual Members belonging to an EPS member society is: Sw.Fr. 44.-; independent members: Sw.Fr. 132.members of a Collaborating Society: Sw.Fr. 55.

\section{Editor: E.N. Shaw}

Editorial Board:

A. Baratoff, F. James, M. Lehmann,

M. Mayor, J. Muller, M. Siegrist

Editorial and Advertising Office at the EPS Secretariat

Address: EUROPEAN PHYSICAL SOCIETY P. O. Box 69 . CH-1213 Petit-Lancy 2

Switzerland

Telephone: Geneva (22) 931130

Telex: 428024 eps ch

Cables: europhys genève

Printed by: Pfirter frères sa CH-1213 Petit-Lancy/Switzerland 